\title{
Nonlinear saturation of baroclinic instability: part I: the two-layer model
}

Article

Published Version

Shepherd, T. G. (1988) Nonlinear saturation of baroclinic instability: part I: the two-layer model. Journal of the Atmospheric Sciences, 45 (14). pp. 2014-2025. ISSN 15200469 doi: https://doi.org/10.1175/15200469(1988)045<2014:NSOBIP>2.0.CO;2 Available at https://centaur.reading.ac.uk/32906/

It is advisable to refer to the publisher's version if you intend to cite from the work. See Guidance on citing.

Published version at: http://dx.doi.org/10.1175/1520-0469(1988)045<2014:NSOBIP>2.0.CO;2

To link to this article DOI: http://dx.doi.org/10.1175/1520-

0469(1988)045<2014:NSOBIP>2.0.CO;2

Publisher: American Meteorological Society

All outputs in CentAUR are protected by Intellectual Property Rights law, including copyright law. Copyright and IPR is retained by the creators or other copyright holders. Terms and conditions for use of this material are defined in the End User Agreement.

www.reading.ac.uk/centaur

\section{CentAUR}


Central Archive at the University of Reading

Reading's research outputs online 


\title{
Nonlinear Saturation of Baroclinic Instability. Part I: The Two-Layer Model
}

\author{
THEODORE G. SHEPHERD \\ Department of Applied Mathematics and Theoretical Physics, University of Cambridge, Silver Street, Cambridge, United Kingdom
}

(Manuscript received 4 August 1987, in final form 22 December 1987)

\begin{abstract}
A rigorous bound is derived which limits the finite-amplitude growth of arbitrary nonzonal disturbances to an unstable baroctinic zonal flow within the context of the two-layer model. The bound is valid for conservative (unforced) flow, as well as for forced-dissipative flow that when the dissipation is proportional to the potential vorticity. The method used to derive the bound relies on the existence of a nonlinear Liapunov (normed) stability theorem for subcritical flows, which is a finite-amplitude generalization of the Charney-Stern theorem.

For the special case of the Phillips model of baroclinic instability, and in the limit of infinitesimal initial nonzonal disturbance amplitude, an improved form of the bound is possible which states that the potential enstrophy of the nonzonal flow cannot exceed $\epsilon \beta^{2} / 3$, where $\epsilon=\left(U-U_{\text {crit }}\right) / U_{\text {crit }}$ is the (relative) supercriticality. This upper bound turns out to be extremely similar to the maximum predicted by the weakly nonlinear theory. For unforced flow with $\epsilon<1$, the bound demonstrates that the nonzonal flow cannot contain all of the potential enstrophy in the system; hence in this range of initial supercriticality the total flow must remain, in a certain sense, "close" to a zonal state.
\end{abstract}

\section{Introduction}

Ever since the pioneering work of Charney (1947) and Eady (1949), the theory of baroclinic instability has occupied a prominent position in dynamical meteorology. These early studies demonstrated that the existence of synoptic-scale cyclone waves in the atmosphere could be explained in terms of the linear instability of a baroclinic zonal flow to wavy disturbances. The linear theory has since been developed by many workers, too numerous to mention here. As with all linear instability theories, however, the results of these studies are only valid so long as the wave amplitude remains sufficiently small, and they must eventually break down.

A crucial question therefore concerns the ultimate equilibration of baroclinic instabilities and the determination of the maximum wave amplitude. Beginning with Pedlosky (1970) and Drazin (1970) these matters have been addressed within the context of weakly nonlinear theory. Such theory provides detailed information on initial wave saturation and on whether the equilibrated state is steady or oscillatory. Apart from the formidable algebraic complexity of this approach, however, the results are subject to significant restrictions. In particular, the unstable flow must be only slightly supercritical. Because the perturbation expansions which are employed are asymptotic rather than

Corresponding author address: Dr. Theodore G. Shepherd, Dept. of Physics, University of Toronto, Toronto M5 SIA7 Canada. convergent, one must ultimately resort to numerical calculation in order to determine the range of validity of the solutions. Perhaps most seriously, these weakly nonlinear theories implicitly enforce spatial (i.e. modal) truncations by their choice of initial conditions, thus precluding "inter alia"-the irreversible spectral cascades that will generally occur in a system with an infinite number of degrees of freedom. Klein and Pedlosky (1986) have recently found in this context that at $O$ (1) supercriticality, severely truncated systems give qualitatively unreliable results; cf. also Mak (1985).

An alternative approach for treating the nonlinear dynamics of baroclinic flow is provided by geostrophic turbulence theory (Charney 1971; Rhines 1977; Salmon 1978; Herring 1980). In such theory the flow is usually presumed to develop into a state of horizontal and (rescaled) vertical homogeneity and isotropy, a presumption which effectively amounts to an assumption of randomization or dynamical ergodicity. (In a channel, of course, geostrophic turbulence theory would have to take account of the fact that the boundary conditions preclude a complete breakup of the zonal flow, and this is a nontrivial matter. But in doubly-periodic geometry, to which the results of this paper equally apply, one could choose a frame of reference with zero net zonal momentum, and then the turbulence arguments described above apply directly.) However, it has recently been shown (Shepherd 1987) that two-dimensional flow on a beta-plane is provably nonergodic for sufficiently small wave steepness, and that the arguments of turbulence theory must therefore be used with caution in that problem. The ergodic hypothesis is, after all, only a hypothesis, and is not uni- 
versally valid. The possibility exists that a similar result might hold with respect to baroclinic flow, namely that an unstable zonal flow with sufficiently small supercriticality might be prevented from breaking up into geostrophic turbulence.

This paper addresses the question of finite-amplitude saturation within a fully nonlinear context. The idea is disarmingly simple, but apparently quite powerful, and relies on the existence of a nonlinear Liapunov (normed) stability theorem for disturbances to a baroclinic zonal basic flow. It may seem odd that a stability theorem for subcritical flow can constrain the behavior of supercritical flow, but such is indeed the case. The point is that a (possibly infinitesimal) wavy disturbance to a supercritical zonal flow may be regarded as a finiteamplitude disturbance (including a zonal-mean component) to a subcritical basic flow, and is then constrained by the nonlinear stability theorem. By minimizing the bound over the class of subcritical flows, a rigorous bound on the amplitude of the wavy part of the flow can be obtained.

In this study, the method outlined above is applied to the problem of baroclinic instability in a two-layer fluid. The governing equations for conservative flow are reviewed in section 2 , and the generalized CharneyStern theorem for this system derived in section 3 . In section 4 the theorem is used to obtain a rigorous bound on the growth of wavy disturbances to an unstable flow. The results are applied to the Phillips (1954) model of baroclinic instability in section 5 ; in the special case of an initially infinitesimal wavy disturbance, the tightest bound takes the form (5.5). It is also shown that for a certain choice of a constant of integration, it is possible for the zonal flow to satisfy the boundary conditions while having zero potential enstrophy; therefore, all of the potential enstrophy is available, at least in principle, to the eddies. In section 6 , the generalized Charney-Stern theorem and the resulting nonlinear saturation bounds are shown to apply to forceddissipative flow in the case where the dissipation is proportional to the potential vorticity in section 6 . A comparison of the bound (5.5) with the predictions of weakly nonlinear theory for single-wave equilibration is made in section 7, and the agreement found to be quite close. In a final section, some implications of these results are considered. The case of continuously stratified flow will be treated in Part II of this study.

\section{Governing equations for the two-layer model}

Perhaps the simplest system exhibiting the phenomenon of baroclinic instability is that of the two-layer model, considered first by Phillips (1954). A thorough treatment of the system can be found in Pedlosky (1979 $\$ 6.17, \$ 7.11)$, whose notation is largely followed here. The flow is governed by conservation of quasigeostrophic potential vorticity in each layer, namely

$$
\frac{D_{i} P_{i}}{D t}=0, \quad i=1,2 \text {, }
$$

i.e.

$$
\frac{\partial P_{i}}{\partial t}+J\left(\Phi_{i}, P_{i}\right) \equiv \frac{\partial P_{i}}{\partial t}+\frac{\partial \Phi_{i}}{\partial x} \frac{\partial P_{i}}{\partial y}-\frac{\partial \Phi_{i}}{\partial y} \frac{\partial P_{i}}{\partial x}=0,
$$

where $i=1$ and $i=2$ refer to the upper and lower layers, respectively, $\Phi_{i}$ is the geostrophic streamfunction, and

$$
P_{i} \equiv \nabla^{2} \Phi_{i}+\beta y+(-1)^{i} F\left(\Phi_{1}-\Phi_{2}\right) .
$$

Here $\nabla^{2}$ is the two-dimensional Laplacian operator, $\beta$ the (linear) gradient of the Coriolis parameter, and $F$ the internal rotational Froude number (a measure of the static stability of the system); equal layer depths are assumed. The domain is infinite in the zonal coordinate $x$ but bounded in the meridional coordinate $y$, with boundary conditions

$$
\frac{\partial \Phi_{i}}{\partial x}=0, \quad \frac{\partial^{2} \bar{\Phi}_{i}}{\partial y \partial t}=0 \quad \text { at } \quad y=0,1
$$

where the overbar refers to a zonal average

$$
\bar{f} \equiv \lim _{X \rightarrow \infty} \frac{1}{2 X} \int_{-X}^{+X} f(x) d x
$$

The system described by (2.1) possesses certain integral invariants. It is straightforward to check that the integral of (kinetic plus available potential) energy,

$$
E \equiv \frac{1}{2}\left\{\left|\nabla \Phi_{1}\right|^{2}+\left|\nabla \Phi_{2}\right|^{2}+F\left(\Phi_{1}-\Phi_{2}\right)^{2}\right\}
$$

is conserved in time, and it is obvious that any function of $P_{1}$ and $P_{2}$ is likewise conserved. Less obviously, perhaps, the integral of what we shall (following the usage of Charney 1971) call the potential enstrophy

$$
\begin{aligned}
& Z \equiv \frac{1}{2}\left\{\left(P_{1}-\beta y\right)^{2}+\left(P_{2}-\beta y\right)^{2}\right\} \\
& =\frac{1}{2}\left\{\left(\nabla^{2} \Phi_{1}\right)^{2}+\left(\nabla^{2} \Phi_{2}\right)^{2}\right\}+F^{2}\left(\Phi_{1}-\Phi_{2}\right)^{2} \\
& +F\left(\Phi_{2}-\Phi_{1}\right)\left(\nabla^{2} \Phi_{1}-\nabla^{2} \Phi_{2}\right)
\end{aligned}
$$

is also a global invariant in this geometry. Conservation of $Z$ follows from the relation

$$
\begin{aligned}
& \int \frac{1}{2}\left(\overline{P_{1}^{2}}+\overline{P_{2}^{2}}\right) d y=\int \bar{Z} d y \\
& \quad+\beta \int y\left(\nabla^{2} \bar{\Phi}_{1}+\nabla^{2} \bar{\Phi}_{2}\right) d y+\beta^{2} \int y^{2} d y
\end{aligned}
$$

because the last term of (2.6) is a constant of the geometry, while the penultimate is just $\beta$ times Kelvin's impulse, which is separately conserved in the zonallysymmetric problem considered here. ${ }^{1}$

\footnotetext{
'In a closed geometry, of course, conservation of Kelvin's impulse would be lost because of the broken zonal symmetry, and so the potential enstrophy would not be a conserved quantity.
} 
Now introduce a basic state $(\Phi, P)=(\Psi, Q)$ consisting of a steady zonal flow $U_{i}(y)=-d \Psi_{i} / d y$, with associated potential-vorticity gradients

$$
\frac{d Q_{i}}{d y} \equiv Q_{i}^{\prime}(y)=-\frac{d^{2} U_{i}}{d y^{2}}+\beta-(-1)^{i} F\left(U_{1}-U_{2}\right)
$$

It is well known that a sufficient condition for linear stability of the basic state is that the gradients (2.7) both have the same sign; for a given flow this must occur for sufficiently large $\beta$, in which case the basic state is referred to as 'subcritical'. If we now consider the total flow to consist of this basic state plus a disturbance $(\psi, q)$, viz.

$$
\Phi_{i}=\Psi_{i}+\psi_{i}, \quad P_{i}=Q_{i}+q_{i},
$$

with

$$
q_{i}=\nabla^{2} \psi_{i}+(-1)^{i} F\left(\psi_{1}-\psi_{2}\right),
$$

then noting that the basic flow (being zonal) is itself an exact steady solution to (2.1) it follows that the disturbance potential vorticity is governed by the exact nonlinear equation

$$
\frac{D_{i} q_{i}}{D t}=-J\left(\psi_{i}, Q_{i}\right)=-\frac{\partial \psi_{i}}{\partial x} \frac{d Q_{i}}{d y}=-\frac{D_{i} Q_{i}}{D t}
$$

in each layer $i=1,2$.

\section{The finite-amplitude generalized Charney-Stern theorem}

The method presented in this paper for bounding the growth of disturbances to a supercritical baroclinic flow relies on the existence of a finite-amplitude Liapunov stability theorem for disturbances to a subcritical basic flow, stated as follows: the (disturbance) potential enstrophy at any time $t$ is bounded in terms of its value at $t=0$ according to

$$
\begin{aligned}
\int \frac{1}{2}\left\{\overline{q_{1}^{2}}\right. & \left.+\overline{q_{2}^{2}}\right\}(t) d y \\
& \leqslant \frac{\left|Q_{i}^{\prime}(y)\right|_{\max }}{\left|Q_{i}^{\prime}(y)\right|_{\min }} \int \frac{1}{2}\left\{\overline{q_{1}^{2}}+\overline{q_{2}^{2}}\right\}(0) d y,
\end{aligned}
$$

valid whenever the basic-state potential-vorticity gradients (2.7) both have the same sign. Equation (3.1) is in effect a statement of Liapunov (normed) stability, the norm in this case being just the square root of the disturbance potential enstrophy. It may be considered the finite-amplitude generalization of the CharneyStern stability theorem, and is related to the generalized Rayleigh theorem recently derived by McIntyre and Shepherd [1987, Eq. (6.28)] and used by Shepherd (1987) to examine the ergodicity of two-dimensional flow on a beta-plane. The essential method leading to (3.1) is originally due to Arnol'd (1966), who used it in the context of two-dimensional nonparallel flow to derive his two nonlinear stability theorems. A general discussion of Arnol'd's method together with a com- pendium of applications is provided by Holm et al. (1985) and Abarbanel et al. (1986).

Although the proof of (3.1) may be inferred from the previous papers referred to above, the direct proof for the two-layer model is so straightforward that, for the sake of completeness, it is worth presenting here. Moreover, some of the intermediate steps will be required when the forced-dissipative case is considered in section 6 .

We start with a zonal basic state $(\Psi, Q)$ and disturbance $(\psi, q)$ as defined in section 2 , and assume that $Q_{i}^{\prime}(y)$ are of definite sign. The $x$-invariance of the basic state $Q_{i}(y)$ allows the definition of the inverse function $Y_{i}(Q)$. Now introduce the second-order disturbance quantity

$$
A_{i}(Q, q) \equiv-\int_{0}^{q}\left\{Y_{i}(Q+\tilde{q})-Y_{i}(Q)\right\} d \tilde{q},
$$

which is of definite sign because of the hypothesis on $Q_{i}^{\prime}(y)$. Note that in the limit of small disturbance amplitude,

$$
A_{i}(Q, q) \approx-\frac{1}{2} Y_{i}^{\prime}(Q) q^{2}=-\frac{1}{2} \frac{q^{2}}{Q_{i}^{\prime}(y)},
$$

which is just (the negative of) the linearized "EliassenPalm wave activity" (Andrews and McIntyre 1976; Held 1985). It may be verified that

$$
\begin{gathered}
\frac{\partial A_{i}}{\partial Q}=-Y_{i}(Q+q)+Y_{i}(Q)+q Y_{i}^{\prime}(Q) \\
\frac{\partial A_{i}}{\partial q}=-Y_{i}(Q+q)+Y_{i}(Q),
\end{gathered}
$$

and thence, using (2.10), that

$$
\begin{aligned}
\frac{D_{i}}{D t} A_{i}\left(Q_{i}, q_{i}\right)= & \frac{\partial A_{i}}{\partial Q_{i}} \frac{D_{i} Q_{i}}{D t}+\frac{\partial A_{i}}{\partial q_{i}} \frac{D_{i} q_{i}}{D t} \\
= & q_{i} Y_{i}^{\prime}(Q) \frac{\partial \psi_{i}}{\partial x} Q_{i}^{\prime}(y) \\
= & \frac{1}{2} \frac{\partial}{\partial x}\left\{\left\{\frac{\partial \psi_{i}}{\partial x}\right\}^{2}-\left\{\frac{\partial \psi_{i}}{\partial y}\right\}^{2}-F \psi_{i}^{2}\right\} \\
& +\frac{\partial}{\partial y}\left\{\frac{\partial \psi_{i}}{\partial x} \frac{\partial \psi_{i}}{\partial y}\right\}+F \psi_{j} \frac{\partial \psi_{i}}{\partial x},
\end{aligned}
$$

with the last step involving the use of (2.9) and integration by parts, and the subscript $j=1$ when $i=2$, and vice versa. When averaged in $x$, this is just the twolayer quasi-geostrophic version of the generalized Eliassen-Palm theorem of Killworth and McIntyre [1985, Eq. (5.17)]. The flux divergence terms on the right-hand side of (3.4) vanish under a zonal average and integration across the channel, using (2.3). Then adding the equations for both layers together, the remaining two terms on the right-hand side can be grouped together into a term $\partial\left(F \psi_{1} \psi_{2}\right) / \partial x$, which sim- 
ilarly vanishes under a zonal average. One thus arrives at the global conservation law

$$
\frac{d}{d t} \int\left\{\overline{A_{1}\left(Q_{1}, q_{1}\right)}+\overline{A_{2}\left(Q_{2}, q_{2}\right)}\right\} d y=0 .
$$

One may consider the integrand of (3.5) to be the density of a pseudomomentum; see McIntyre and Shepherd $(1987,87)$ for a discussion of how such a conservation law is related to the symmetry properties of the basic state.

To complete the derivation, it suffices to note the inequalities

$$
\begin{aligned}
\frac{\frac{1}{2} q^{2}}{\left|Q_{i}^{\prime}(y)\right|_{\max }} & =\int_{0}^{q}\left|Y_{i}^{\prime}(Q)\right|_{\min } \tilde{q} d \tilde{q} \leqslant\left|A_{i}(Q, q)\right| \\
& \leqslant \int_{0}^{q}\left|Y_{i}^{\prime}(Q)\right|_{\max } \tilde{q} d \tilde{q}=\frac{\frac{1}{2} q^{2}}{\left|Q_{i}^{\prime}(y)\right|_{\min }},
\end{aligned}
$$

the min and the max being taken over all values of $y$ and over both layers, from which (3.1) follows directly.

\section{Finite-amplitude saturation of unforced baroclinic instability}

We are now in a position to address the main problem at issue in this paper. Given an arbitrary nonzonal disturbance to an initially supercritical (i.e., unstable) zonal flow, can a bound be placed on the ultimate nonlinear growth of the disturbance? Obviously the growth is constrained by the total energy and potential enstrophy in the system, but by using the result (3.1) it turns out that the growth is in fact limited by a smaller quantity, which for initially infinitesimal wavy disturbances goes to zero in the limit of small supercriticality. The proof now follows.

Consider an initial condition consisting of a zonal flow

$$
-\frac{d \bar{\Phi}_{1}}{d y}=\frac{\beta}{F}(1+\epsilon) g(y), \quad-\frac{d \bar{\Phi}_{2}}{d y}=0,
$$

plus an arbitrary nonzonal disturbance with streamfunction $\phi_{i}$. We take $g$ positive with a maximum of unity (to give a westerly jet), and presume that $g^{\prime \prime}(y)$ $<F /(1+\epsilon)$ so that the initial zonal flow is barotropically stable (in the sense that $d \bar{P}_{i} / d y$ is single-signed within each layer). This is certainly not the most general class of flows possible but it does cover the regime of most interest in the present context. (A modification of the derivation for nonzero flow in the lower layer would be straightforward.) Note that $d \bar{P}_{1} / d y$ is always positive (provided $\epsilon>-1$ ), and that $d \bar{P}_{2} / d y$ is positive when $\epsilon<0$, but negative at the jet $\max$ when $\epsilon>0$. We are of course interested in the supercritical case, $\epsilon$ $>0$, and refer to $\epsilon$ as the (relative) supercriticality.

Now introduce a subcritical basic flow

$$
U_{1}=\frac{\beta}{F}(1-\delta) g(y), \quad U_{2}=0 .
$$

Nothing is lost by restricting attention to $U_{1} \geqslant 0$, so $\delta$ $\leqslant 1$. The initial flow may be considered as a finiteamplitude disturbance to this basic flow, the disturbance consisting of a wavy part $\psi_{i}^{\prime}=\phi_{i}$ (the departure from the zonal mean) together with a zonal-mean part

$$
\bar{\psi}_{1}=-\frac{\beta}{F}(\epsilon+\delta) G(y), \quad \bar{\psi}_{2}=0
$$

[the integral of minus the difference between (4.1) and (4.2)], where $G(y)=\int g(y) d y+\lambda$, and $\lambda$ is an (as yet arbitrary) constant of integration. This finite-amplitude disturbance to the stable basic flow (4.2) has potential vorticity $q_{i}$ given at $t=0$ by

$$
\begin{aligned}
q_{1}(t=0)= & \nabla^{2} \phi_{1}+F\left(\phi_{2}-\phi_{1}\right) \\
& +\beta(\epsilon+\delta) G(y)-\frac{\beta}{F}(\epsilon+\delta) g^{\prime}(y), \\
q_{2}(t=0)= & \nabla^{2} \phi_{2}+F\left(\phi_{1}-\phi_{2}\right)-\beta(\epsilon+\delta) G(y),
\end{aligned}
$$

and the generalized Charney-Stern theorem (3.1) evidently places a constraint on the possible growth of $q_{i}$ with time. In fact, we are particularly interested in bounding the wavy (or nonzonal) part of the disturbance, $q_{i}^{\prime}$, but this is obviously bounded by (3.1) as well since

$$
\int \overline{q_{i}^{\prime 2}} d y \leqslant \int \overline{q_{i}^{2}} d y .
$$

The idea is now to choose $\delta$ and $\lambda$ (which are arbitrary) in such a way as to obtain the tightest constraint.

For the basic flow (4.2), we have

$$
\begin{gathered}
\frac{d Q_{1}}{d y}=\beta+\beta(1-\delta) g(y)-\frac{\beta}{F}(1-\delta) g^{\prime \prime}(y), \\
\frac{d Q_{2}}{d y}=\beta-\beta(1-\delta) g(y),
\end{gathered}
$$

from which it follows that

$$
\begin{gathered}
\left|Q_{i}^{\prime}(y)\right|_{\max }=\beta\{2-\delta+\gamma(1-\delta)\}, \\
\left|Q_{i}^{\prime}(y)\right|_{\min }=\beta \delta,
\end{gathered}
$$

with $\gamma \equiv F^{-1} \max \left\{-g^{\prime \prime}(y)\right\} \geqslant 0 .{ }^{2}$ Using (4.3), the initial disturbance potential enstrophy may be written as

$$
\int_{0}^{1} \frac{1}{2}\left\{\overline{q_{1}^{2}}+\overline{q_{2}^{2}}\right\}(0) d y=\beta^{2} \Lambda(\lambda)(\epsilon+\delta)^{2}+\hat{z}_{0},
$$

where

$$
\begin{aligned}
\Lambda(\lambda) \equiv \int_{0}^{1} G^{2} d y+\frac{1}{2} F^{-2} \int_{0}^{1} g^{\prime 2} d y & \\
& \quad-F^{-1} \int_{0}^{1} G g^{\prime} d y
\end{aligned}
$$

\footnotetext{
${ }^{2}$ There is an implicit (but reasonable) presumption in (4.5a) that $-g^{\prime \prime}(y)$ takes its maximum where $g(y)$ does.
} 
and

$$
\begin{aligned}
& \hat{z}_{0} \equiv \int_{0}^{1} \frac{1}{2}\left\{\overline{\left\{\nabla^{2} \phi_{1}\right.}+F\left(\phi_{2}-\phi_{1}\right)\right\}^{2} \\
&\left.+\overline{\left\{\nabla^{2} \phi_{2}+F\left(\phi_{1}-\phi_{2}\right)\right\}^{2}}\right\} d y
\end{aligned}
$$

is the potential enistrophy of the nonzonal flow at $t$ $=0$. We now take advantage of the arbitrariness of the constant of integration $\lambda$, and choose $\lambda$ so as to minimize $\Lambda(\lambda)$ in (4.7). The point is that the ultimate goal is an upper bound on the potential enstrophy of the nonzonal part of the flow, which is independent of $\lambda$; in general, however, the boundary conditions (2.3) require that the potential enstrophy of the zonal flow can never vanish, so it is advantageous to choose $\lambda$ (which is equivalent to redefining the origin) in such a way as to minimize the amount of potential enstrophy which is unavailable to the eddies. (There is a helpful analogy here in that while the total kinetic energy of the system is dependent on the frame of reference, one is free to perform one's analysis in any frame of reference because of the Galilean-invariance of the underlying dynamics.) Setting

$$
\Lambda \equiv \min _{\lambda} \Lambda(\lambda)
$$

and defining $\hat{z}_{0}=\beta^{2} \Lambda z_{0}$, the initial disturbance potential enstrophy (4.6) becomes

$$
\int_{0}^{1} \frac{1}{2}\left\{\overline{q_{1}^{2}}+\overline{q_{2}^{2}}\right\}(0) d y=\beta^{2} \Lambda\left\{(\epsilon+\delta)^{2}+z_{0}\right\} \text {. }
$$

Using (4.5) and (4.9), the right-hand side of (3.1), providing the bound on the enstrophy of the nonzonal part of the flow, then takes the form

$$
\begin{aligned}
\beta^{2} \Lambda \frac{1}{\delta}(2-\delta+\gamma(1-\delta)) & \left\{(\epsilon+\delta)^{2}+z_{0}\right\} \\
& \equiv \beta^{2} \Lambda f\left(\delta, \epsilon, \gamma, z_{0}\right) .
\end{aligned}
$$

For given $\epsilon, \gamma$ and $z_{0}$, we seek the minimum of $f(\delta, \epsilon$, $\gamma, z_{0}$ ) over the interval $0<\delta \leqslant 1$. Noting that $f$ diverges as $\delta \rightarrow 0$, this minimum will either be a local minimum, attained at some $\delta_{\min }<1$, or it will be attained at $\delta$ $=1$; but in the latter case the bound is equal to the total amount of potential enstrophy in the system, namely

$$
\int \bar{Z} d y=\beta^{2} \Lambda\left\{(1+\epsilon)^{2}+z_{0}\right\}
$$

Seeking the local minimum, setting $\partial f / \partial \delta=0$ leads to the cubic equation

$\delta^{3}+\left\{\epsilon-\frac{(2+\gamma)}{2(1+\gamma)}\right\} \delta^{2}+\frac{(2+\gamma)}{2(1+\gamma)}\left(\epsilon^{2}+z_{0}\right)=0$

For sufficiently small $z_{0}(4.11)$ has three real roots, and the minimum of $f$ is attained at

$$
\delta_{\min }=2 \rho \cos ((\phi+4 \pi) / 3)-\rho \text {, }
$$

where

$$
\begin{aligned}
\rho & \equiv \frac{1}{3}\left\{\epsilon-\frac{(2+\gamma)}{2(1+\gamma)}\right\}, \\
\cos \phi & \equiv-\frac{27}{4} \frac{(2+\gamma)}{(1+\gamma)}\left\{\epsilon-\frac{(2+\gamma)}{2(1+\gamma)}\right\}^{-3}\left\{\epsilon^{2}+z_{0}\right\}-1
\end{aligned}
$$

(see Bronshtein and Semendyayev, 1985, §2.4.2.3). Figure 1 shows $f\left(\delta_{\min }, \epsilon, \dot{\gamma}, z_{0}\right)$ as a function of $\epsilon$, for various values of $z_{0}$, for the two cases $\gamma=0$ and $\gamma$ $=1$. The total amount of potential enstrophy in the system is also indicated.

An important special case is that where the initial wavy disturbance has infinitesimal amplitude, viz. the limit $z_{0} \rightarrow 0$. In that limit the cubic (4.11) is easily seen to factor to

$$
\begin{aligned}
& (\delta+\epsilon)\left\{\delta^{2^{\prime}}-\frac{(2+\gamma)}{2(1+\gamma)} \delta+\frac{(2+\gamma)}{2(1+\gamma)} \epsilon\right\}=0 \\
& \delta_{\min }=\frac{(2+\gamma)}{4(1+\gamma)}\left\{1-\left(1-\frac{8(1+\gamma) \epsilon}{(2+\gamma)}\right)^{1 / 2}\right\} .
\end{aligned}
$$

It is evident from (4.14) that for sufficiently small $\epsilon$, $\delta_{\text {min }}$ is well approximated as $\delta_{\min } \approx \epsilon$. In fact, numerical calculation reveals that in using $\delta=\epsilon$ the true minimum of $f$ is overestimated by no more than about $3 \%$ over the useful range of $\epsilon$. The latter is the range for which the upper bound on the wavy potential enstrophy is less than $\int \bar{Z} d y$, namely $f\left(\delta_{\min }, \epsilon, \gamma, 0\right)<(1+\epsilon)^{2}$; approximating $f\left(\delta_{\min }, \epsilon, \gamma, 0\right)$ by $f(\epsilon, \epsilon, \gamma, 0)$ the condition on $\epsilon$ becomes

$$
\begin{aligned}
4 \epsilon(2-\epsilon & +\gamma(1-\epsilon))<(1+\epsilon)^{2} \\
& \Leftrightarrow(5+4 \gamma) \epsilon^{2}-2(3+2 \gamma) \epsilon+1>0,
\end{aligned}
$$

which is satisfied for

$$
\epsilon<\frac{1}{5+4 \gamma}
$$

One may therefore write down the general bound

$$
\int \frac{1}{2}\left\{\overline{q_{1}^{\prime 2}}+\overline{q_{2}^{\prime 2}}\right\} d y \leqslant \beta^{2} \Lambda f(\gamma, \epsilon),
$$

valid in the limit $z_{0} \rightarrow 0$, where

$$
\begin{aligned}
f(\gamma, \epsilon) & \equiv f\left(=\epsilon, \epsilon, \gamma, z_{0}=0\right) \\
& = \begin{cases}4 \epsilon[2-\epsilon+\gamma(1-\epsilon)], & \epsilon \leqslant(5+4 \gamma)^{-1} \\
(1+\epsilon)^{2}, & \epsilon \geqslant(5+4 \gamma)^{-1}\end{cases}
\end{aligned}
$$

Equation (4.17) represents a rigorous upper bound on the possible nonlinear growth of an infinitesimal wavy disturbance to an unstable zonal flow (4.1) with supercriticality $\epsilon$.

For a given supercritical flow, taking the limit $\beta \rightarrow$ 0 also requires that $\epsilon \rightarrow \infty$; so in this limit the stability theorem ceases to usefully constrain the flow evolution. 

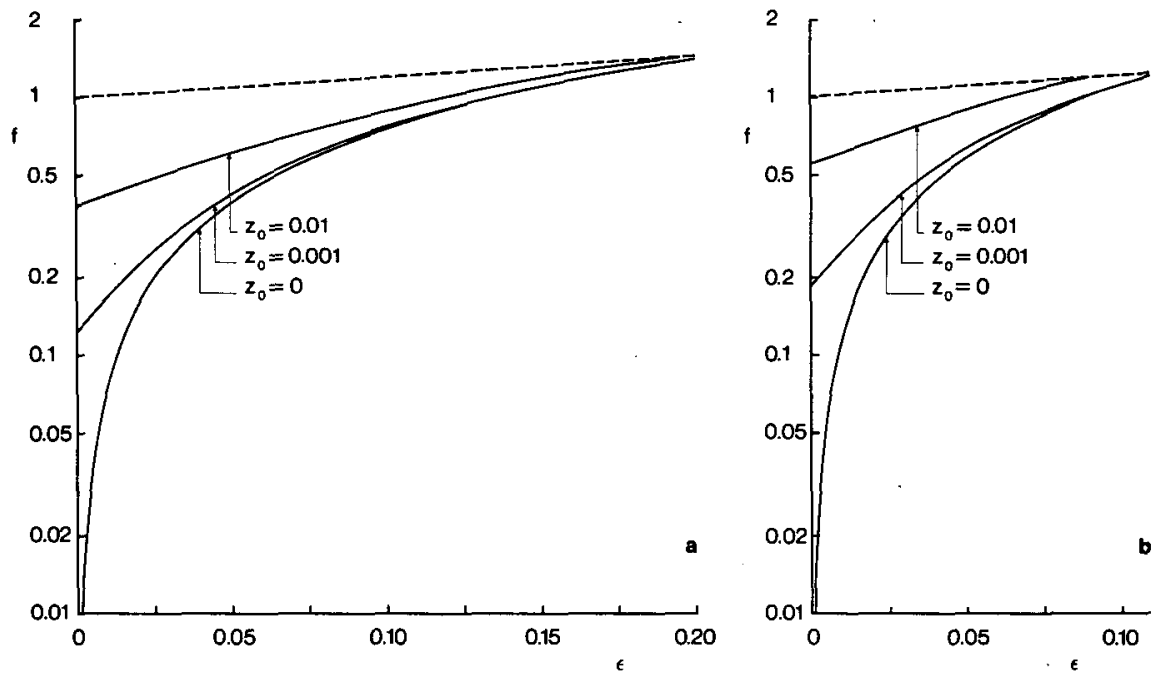

Fig. 1. (a) The solid curves show the graph of $f\left(\delta_{\min }, \epsilon, \gamma, z_{0}\right)$ versus $\epsilon$ for $\gamma=0$ and for various $z_{0}$, as indicated. The upper dashed curve shows $f\left(1, \epsilon, \gamma, z_{0}\right)$, which represents the value of $f$ corresponding to the total amount of potential enstrophy in the system: (b) Same as in (a), except for $\gamma=1$.

\section{Application to the Phillips model}

Attention is now restricted to the Phillips (1954) model of baroclinic instability, for which the initial zonal flow has no meridional shear, and the zonalmean potential vorticity gradients are therefore independent of $y$ (at least at $t=0$ ). This is a special case of the class of flows considered above, with $g(y) \equiv 1, G(y)$ $=y+\lambda, \gamma=0$, and $\Lambda=1 / 12$ for the choice $\lambda=-1 / 2$. The bounds for different $z_{0}$ given by (4.10) with (4.12), using these values, therefore apply and are shown in Fig. 1a. In the limit of an infinitesimal initial wavy disturbance, $z_{0} \rightarrow 0$, the bound (4.17) takes the form:

$$
\int \frac{1}{2}\left\{\overline{q_{1}^{\prime 2}}+\overline{q_{2}^{\prime 2}}\right\} d y \leqslant \frac{1}{12} \beta^{2} f(\epsilon)
$$

where

$$
f(\epsilon) \equiv f(\gamma=0, \epsilon)= \begin{cases}4 \epsilon(2-\epsilon), & \epsilon \leqslant 0.2 \\ (1+\epsilon)^{2}, & \epsilon \geqslant 0.2 .\end{cases}
$$

I. M. Held (personal communication, 1988) has pointed out that the bound (5.1), (5.2) may be improved upon by using, in place of (3.1),

$$
\begin{aligned}
\int \frac{1}{2}\left\{\overline{q_{1}^{2}}+\overline{q_{2}^{2}}\right\} & (t) d y \leqslant\left|Q_{i}^{\prime}(y)\right|_{\max } \mathcal{A}(t) \\
& =\left|Q_{i}^{\prime}(y)\right|_{\max } \mathcal{A}(0) \\
& =\int \frac{1}{2}\left\{\overline{q_{1}^{2}}+\frac{Q_{1}^{\prime}(y)}{Q_{2}^{\prime}(y)} \overline{q_{2}^{2}}\right\}(0) d y .
\end{aligned}
$$

Here $\mathcal{A}(t)$ is the absolute value of the integrated pseudomomentum, which for the special case of constant $Q_{i}^{\prime}(y)$ is given by (6.5) below [and thereby leads to (5.3b)]. This will evidently yield a tighter bound than will (3.1). For the Phillips model in the limit $z_{0} \rightarrow 0$, the right-hand side of $(5.3 \mathrm{~b})$ takes the form

$$
\left\{1+\frac{2-\delta}{\delta}\right\} \frac{\beta^{2}}{24}(\epsilon+\delta)^{2}=\frac{\beta^{2}}{12} \frac{(\epsilon+\delta)^{2}}{\delta}
$$

this quantity is minimized (within the interval $0<\delta$ $\leqslant 1$ ) for $\delta=\epsilon$ when $\epsilon \leqslant 1$, and for $\delta=1$ when $\epsilon \geqslant 1$. Therefore, the improved bound may be written

$$
\int \frac{1}{2}\left\{\overline{q_{1}^{12}}+\overline{q_{2}^{\prime 2}}\right\} d y \leqslant \begin{cases}\frac{1}{3} \beta^{2} \epsilon & \text { for } \epsilon \leqslant 1 \\ \frac{1}{12} \beta^{2}(1+\epsilon)^{2} & \text { for } \epsilon \geqslant 1 .\end{cases}
$$

Comparing (5.5) with (5.1), (5.2), it is evident that (5.5) is indeed smaller for $\epsilon<1$, and is in fact twice as small in the limit $\epsilon \rightarrow 0$. This last property could have been anticipated directly by noting that for $\epsilon \rightarrow 0$, $Q_{1}^{\prime}(y) / Q_{2}^{\prime}(y)$ is $\mathrm{O}\left(\epsilon^{-1}\right)$ while $\int \overline{q_{1}^{2}}(0) d y$ and $\int \overline{q_{2}^{2}}(0) d y$ are equal (assuming $z_{0} \rightarrow 0$ ), and thus the right-hand side of (3.1) is, asymptotically, twice as large as that of (5.3b).

It may be instructive to write the bound (5.5) in terms of the initial vertical shear, $U \equiv \beta(1+\epsilon) / F$. This yields

$$
\begin{aligned}
\int \frac{1}{2}\left\{\overline{q_{1}^{\prime 2}}+\overline{q_{2}^{\prime 2}}\right\} d y & \left\{\begin{array}{lll}
\frac{\beta}{3}(U F-\beta) & \text { for } & 1<U F / \beta \leqslant 2 \\
\frac{1}{12} U^{2} F^{2} & \text { for } & U F / \beta \geqslant 2
\end{array}\right.
\end{aligned}
$$


From (5.6) it is clear that in the limit $\beta \rightarrow 0$ the bound simply equals the total amount of potential enstrophy in the system, and the stability theorem is therefore giving no useful information.

To this point, the upper bounds on the wavy potential enstrophy have been compared with $\int \bar{Z} d y$, the total amount of potential enstrophy in the system (which is a constant of the motion). It may, however, be argued that since the boundary conditions (2.3) will not generally permit the zonal flow to vanish, one should really be comparing the bounds with something smaller than $\int \bar{Z} d y$. Indeed, one would ideally like to have a prediction for the wavy potential enstrophy from geostrophic turbulence theory; unfortunately however, the boundary conditions (2.3) make such a calculation rather nontrivial. In the absence of a precise theory, let us make the rash but intuitively plausible hypothesis that turbulence will act to minimize the amount of potential enstrophy residing in the zonal flow. We may then try to determine this minimum, in order to get some idea of how much potential enstrophy is left for the eddies.

It is useful to choose a frame of reference for which the net zonal momentum vanishes (this does not affect $\bar{Z})$. The zonal flow, with streamfunction $\bar{\Phi}_{i}$, must then satisfy the conditions

$$
\begin{aligned}
& \frac{d \bar{\Phi}_{1}}{d y}(0)=-\frac{U}{2}=\frac{d \bar{\Phi}_{1}}{d y}(1), \\
& \frac{d \bar{\Phi}_{2}}{d y}(0)=\frac{U}{2}=\frac{d \bar{\Phi}_{2}}{d y}(1)
\end{aligned}
$$

In fact, there exists a zonal flow, with zero net momentum and satisfying (5.7), which has zero potential enstrophy; viz.

$$
\begin{gathered}
\bar{\Phi}_{1}(y)=\frac{U}{2 \nu}\left(1+e^{-\nu}\right)^{-1}\left\{e^{-v y}-e^{\nu(y-1)}\right\}, \\
\bar{\Phi}_{2}(y)=-\bar{\Phi}_{1}(y),
\end{gathered}
$$

with $\nu \equiv(2 F)^{1 / 2}$. Note that $\bar{\Phi}_{i}(y)=0$ at $y=1 / 2$ (the center of the channel), which corresponds to the choice $\lambda=-1 / 2$ for the Phillips model since then $G(y)$ vanishes at $y=1 / 2$ as well. Thus, for the Phillips model, all of the total potential enstrophy $\beta^{2}(1+\epsilon)^{2} / 12$ is, in principle, available to the eddies, and for $\epsilon<1$ the bound (5.5) is indeed providing a useful constraint on the dynamics.

In the more general case represented by (4.1), it is always possible to find a zonal flow whose baroclinic part has zero potential enstrophy. This is because the baroclinic contribution to $Z$, namely

$$
\frac{1}{4}\left(\nabla^{2} \bar{\tau}\right)^{2}+F^{2} \bar{\tau}^{2}-F \bar{\tau} \nabla^{2} \bar{\tau},
$$

where $\tau \equiv \Phi_{1}-\Phi_{2}$ is the baroclinic streamfunction, will vanish identically for $\bar{\tau}$ of the form

$$
\bar{\tau}(y)=a e^{-\nu y}+b e^{\mu(y-1)},
$$

and the two constants $a$ and $b$ in (5.9) can always be chosen to satisfy the boundary conditions on $d \bar{\tau} / d y$ at $y=0,1$. [The value of $y$ for which (5.9) vanishes will then determine the choice of $\lambda$.] Unlike the case of the Phillips model, however, the barotropic part of $Z$ cannot generally be made to vanish without violating the boundary conditions, and so there will generally be part of the total potential enstrophy which is not available to the eddies.

\section{The forced-dissipative case}

In this section it is shown that the generalized Charney-Stern theorem and the finite-amplitude saturation bounds, derived for conservative flow, hold also for a particular kind of forced-dissipative evolution. Suppose that the dissipation in the flow is proportional to the potential vorticity $P_{i}$, but that the initial unstable flow $P_{i}(t=0)=\bar{P}_{0 i}$ [as given by (4.1) for example] is presumed to be an equilibrium solution. Hence the dissipation is proportional to the disturbance potential vorticity, where the disturbance is, for this purpose, measured relative to the initial unstable flow. In that case the governing equation (2.1) is replaced by

$$
\frac{D_{i} P_{i}}{D t}=-r P_{i}+r
$$

where $r$ is the dissipation coefficient. Although the $-r P_{i}$ term in (6.1) is clearly dissipative, the second term is not so, and can be thought of as a forcing. Indeed, in a steady-state situation the two terms must balance globally. The system given by (6.1) is that studied, for example, by Pedlosky (1982b). Given the level of abstraction of the two-layer model, it is impossible to say which kind of friction is most 'realistic'. However, the more conventional Ekman damping (proportional to $\left.\nabla^{2} \Phi_{i}\right)$ is rather pathological because it distorts the curve of marginal stability (e.g., see Pedlosky 1979, §7.12) and the flow behavior is qualitatively sensitive to the relative strength of the damping coefficients on the two 'lids' (Mak 1987). So (6.1) seems as reasonable a system as any in this context.

The fact that the results carry over in the case of (6.1) is proven in Shepherd $(1988, \$ 4.1)$ in the context of two-dimensional flow and the extension of the proof to the two-layer model is trivial. Rather than duplicating the treatment presented there, we shall here give explicit proof for the special case of the Phillips model, where the basic-state potential-vorticity gradients $Q_{i}^{\prime}(y)$ are independent of $y$ (and positive), and refer the reader to the aforementioned paper for the more general case. 
For the Phillips model, (6.1) takes the form

$$
\begin{aligned}
\frac{D_{i} P_{i}}{D t}=-r P_{i}+r\left\{\beta-(-1)^{i} \beta(1+\epsilon)\right\} y & \\
& +\frac{r}{2}(-1)^{i} \beta(1+\epsilon) ;
\end{aligned}
$$

and then using (6.2) in place of (2.1), (2.10) is replaced by

$$
\frac{D_{i} q_{i}}{D t}=-\frac{\partial \psi_{i}}{\partial x} \frac{d Q_{i}}{d y}-r q_{i}-r(-1)^{i} \beta(\epsilon+\delta)\left(y-\frac{1}{2}\right) .
$$

[Note that the disturbance $q_{i}$ is again defined relative to the subcritical basic state (4.2), as in the previous sections.] In this special case of constant $Q_{i}^{\prime}(y)$, the linearized approximation to the pseudomomentum density, (3.3), is exact at finite amplitude and the pseudomomentum conservation law (3.5) now takes the form

$$
\begin{aligned}
& \frac{d}{d t} \int\left\{\frac{\overline{q_{1}^{2}}}{Q_{1}^{\prime}(y)}+\frac{\overline{q_{2}^{2}}}{Q_{2}^{\prime}(y)}\right\} d y=-2 r \int\left\{\frac{\overline{q_{1}^{2}}}{Q_{1}^{\prime}(y)}+\frac{\overline{q_{2}^{2}}}{Q_{2}^{\prime}(y)}\right\} d y \\
& \quad+2 r \int \beta(\epsilon+\delta)\left\{\frac{\left(y-\frac{1}{2}\right) \overline{q_{1}}}{Q_{1}^{\prime}(y)}-\frac{\left(y-\frac{1}{2}\right) \overline{q_{2}}}{Q_{2}^{\prime}(y)}\right\} d y \quad \text { (6.4) }
\end{aligned}
$$

with the integrand on the left-hand side being the negative of twice the density of pseudomomentum. It is not possible to show that the right-hand side of (6.4) must always be negative. However, we require rather less than this. In order for the saturation bound to hold, it is evidently sufficient that

$$
\begin{aligned}
\mathcal{A}(t) & \equiv \int \frac{1}{2}\left\{\frac{\overline{q_{1}{ }^{2}}}{Q_{1}^{\prime}(y)}+\frac{\overline{q_{2}{ }^{2}}}{Q_{2}^{\prime}(y)}\right\}(t) d y \\
& \leqslant \int \frac{1}{2}\left\{\frac{\overline{q_{1}{ }^{2}}}{Q_{1}^{\prime}(y)}+\frac{\overline{q_{2}{ }^{2}}}{Q_{2}^{\prime}(y)}\right\}(0) d y
\end{aligned}
$$

for any time $t \geqslant 0$. (Note that $\mathcal{A}$ is necessarily positive.)

The proof of (6.5) is by contradiction, as follows. Suppose that (6.5) does not hold, i.e., that $\mathcal{A}(t)>\mathcal{A}(0)$. Then we have (even for finite $\phi_{i}$ )

$$
\begin{aligned}
& \frac{1}{Q_{1}^{\prime}(y)} \int \overline{q_{1}^{2}} d y+\frac{1}{Q_{2}^{\prime}(y)} \int \overline{q_{2}^{2}} d y \\
& \quad>\left\{\frac{1}{Q_{1}^{\prime}(y)}+\frac{1}{Q_{2}^{\prime}(y)}\right\} \int \beta^{2}(\epsilon+\delta)^{2}\left(y-\frac{1}{2}\right)^{2} d y,
\end{aligned}
$$

using (4.3) in this case with $G(y)=y-1 / 2$ and $g^{\prime}(y)$ $\equiv 0$. But

$$
\begin{aligned}
0 \leqslant & \frac{1}{Q_{1}^{\prime}(y)} \int \overline{\left\{q_{1}-\beta(\epsilon+\delta)\left(y-\frac{1}{2}\right)\right\}^{2}} d y \\
& +\frac{1}{Q_{2}^{\prime}(y)} \int \overline{\left\{q_{2}+\beta(\epsilon+\delta)\left(y-\frac{1}{2}\right)\right\}^{2}} d y
\end{aligned}
$$

$$
\begin{aligned}
= & \frac{1}{Q_{1}^{\prime}(y)}\left\{\int \overline{q_{1}^{2}} d y+\int \beta^{2}(\epsilon+\delta)^{2}\left(y-\frac{1}{2}\right)^{2} d y\right. \\
& \left.-2 \int \beta(\epsilon+\delta)\left(y-\frac{1}{2}\right) \overline{q_{1}} d y\right\} \\
& +\frac{1}{Q_{2}^{\prime}(y)}\left\{\int \overline{q_{2}^{2}} d y+\int \beta^{2}(\epsilon+\delta)^{2}\left(y-\frac{1}{2}\right)^{2} d y\right. \\
& \left.+2 \int \beta(\epsilon+\delta)\left(y-\frac{1}{2}\right) \overline{q_{2}} d y\right\} \\
< & \frac{2}{Q_{1}^{\prime}(y)}\left\{\int \overline{q_{1}^{2}} d y-\int \beta(\epsilon+\delta)\left(y-\frac{1}{2}\right) \overline{q_{1}} d y\right\} \\
& +\frac{2}{Q_{2}^{\prime}(y)}\left\{\int \overline{q_{2}^{2}} d y+\int \beta(\epsilon+\delta)\left(y-\frac{1}{2}\right) \overline{q_{2}} d y\right\},
\end{aligned}
$$

using (6.6) to establish the last inequality. This implies that the right-hand side of (6.4) is negative. In particular, the above reasoning shows that $d \mathcal{A} / d t<0$ during the time that $\mathcal{A}$ increased from $\mathcal{A}(0)$ to $\mathcal{A}(t)$, which is a contradiction. Therefore $(6.5)$ holds, from which it follows immediately that the saturation bounds remain valid for the forced-dissipative problem considered here.

\section{Comparison with single-wave equilibration from weakly nonlinear theory}

The weakly nonlinear theory of Pedlosky (1970) provides an explicit prediction of the time evolution of a single-wave disturbance to marginally unstable flow in the Phillips model. It is clearly of interest to see how the maximum wave amplitude predicted by such theory compares with the saturation bounds derived above. As long as the weakly nonlinear theory remains self-consistent, its predictions should be compatible with the fully nonlinear theory described here. This is because the predicted evolution is always a kinematically possible realization of the full system, and the wave-mean interaction is properly accounted for in the weakly nonlinear theory. For simplicity, the discussion here is restricted to the case $z_{0} \rightarrow 0$ of an initially infinitesimal wavy disturbance.

For a given supercritical shear $U$, there is a range of total wavenumbers $\kappa$ which are unstable according to linear theory (see Fig. 2). The supercriticality $\epsilon$ defined by (4.1) represents the (relative) supercriticality with respect to the minimum critical shear $\hat{U}_{c}$. For any $k$ in the unstable range, the absolute supercriticality $\Delta(\kappa)$ is given by

$$
\Delta(\kappa)=U-U_{c}(\kappa),
$$

where the critical shear is

$$
U_{c}(\kappa)=\frac{2 \beta F}{\kappa^{2}\left(4 F^{2}-\kappa^{4}\right)^{1 / 2}} .
$$

It is the square root of this parameter $\Delta(\kappa)$ which is taken as the (small) expansion parameter for a given 


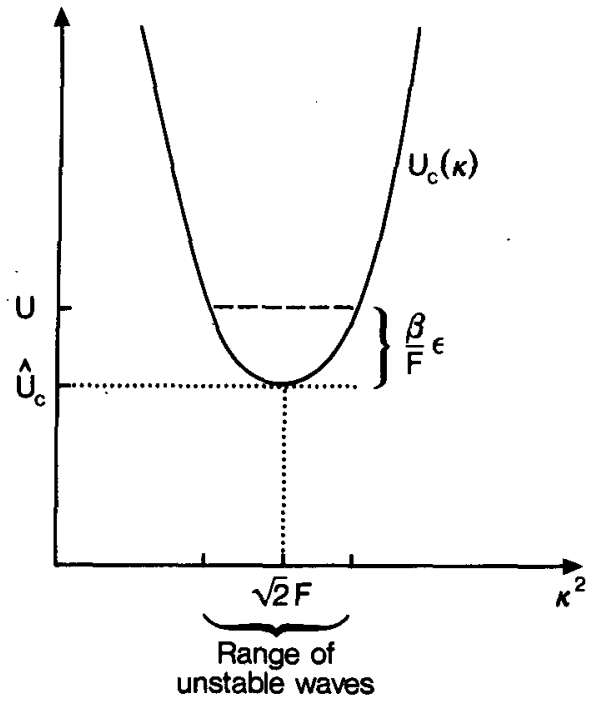

FIG. 2. Schematic of marginal stability curve $U_{C}(\kappa)$, indicating the range of waves which are (linearly) unstable for a given supercriticality $\epsilon$. Instability at minimum critical shear $U=U_{C}$ occurs only at $\kappa^{2}$ $=\sqrt{2} F$.

single wave $\kappa$ in Pedlosky's theory. Note that at $\kappa^{2}$ $=\sqrt{2} F, \Delta(\kappa)=\beta \epsilon / F$ (this is the most unstable wave according to linear theory).

Pedlosky (1970) predicts, for inviscid evolution of an initially infinitesimal disturbance, a maximum wave amplitude of

$$
|A|_{\max }^{2}=\frac{2 c_{0 i}^{2}}{N}
$$

where $\Delta^{1 / 2}|A|$ is the amplitude of the leading order term in the expansion for the wavy part of the upperlayer streamfunction. The $c_{0 i}$ is the imaginary part of the scaled phase speed, with

$$
c_{0 i}^{2}=\frac{2 \beta^{2} F^{2}}{\kappa^{4}\left(\kappa^{2}+2 F\right)^{2}} \frac{\Delta}{|\Delta| U_{c}},
$$

and $N$ is the coefficient of the (stabilizing) cubic term in the Landau equation,

$$
\begin{aligned}
N= & \frac{\left(\beta+F U_{c}\right) U_{c} m^{2} \pi^{2}}{8\left(U_{2}+U_{c}-c\right)^{2}\left(2 m^{2} \pi^{2}+F\right)\left(\kappa^{2}+2 F\right) \kappa^{2}} \\
& \times\left\{\left(\kappa^{2}-F\right)\left(4 m^{2} \pi^{2}+2 F\right)\right. \\
& \left.+\left(2 F^{2}-\kappa^{4}\right)\left\{1+\frac{4 m^{2} \pi^{2} \tanh (F / 2)^{1 / 2}}{(F / 2)^{1 / 2}\left(2 m^{2} \pi^{2}+F\right)}\right\}\right\} .
\end{aligned}
$$

In the above, $m$ is the meridional wavenumber (which must be an integer), and

$$
U_{2}-c=-\frac{U_{c}}{2}+\frac{\beta\left(\kappa^{2}+F\right)}{\kappa^{2}\left(\kappa^{2}+2 F\right)} .
$$

To leading order in $\Delta^{1 / 2}$, the potential vorticity of the wavy part of the flow is given by

$$
\begin{aligned}
& q_{1}^{\prime}=-\frac{\beta+F U_{c}}{U_{2}+U_{c}-c} \Delta^{1 / 2} \operatorname{Re}\left\{A e^{i \theta} \sin (m \pi y)\right\}, \\
& q_{2}^{\prime}=-\frac{\beta-F U_{c}}{U_{2}-c} \Delta^{1 / 2} \operatorname{Re}\left\{A \tilde{\gamma} e^{i \theta} \sin (m \pi y)\right\},(7.7)
\end{aligned}
$$

[Pedlosky 1979, Eq. (7.16.21)], where $\theta$ is the phase and

$$
\tilde{\gamma}=\frac{\kappa^{2}+F}{F}-\frac{\beta+F U_{c}}{F\left(U_{2}+U_{c}-c\right)} .
$$

It follows from (7.3) and (7.7) that the maximum potential enstrophy of the wavy part of the flow, for this weakly nonlinear solution, is to leading order,

$$
\frac{1}{8} \Delta \frac{2 c_{0 i}^{2}}{N}\left\{\left\{\frac{\beta+F U_{c}}{U_{2}+U_{c}-c}\right\}^{2}+\tilde{\gamma}^{2}\left\{\frac{\beta-F U_{c}}{U_{2}-c}\right\}^{2}\right\},
$$

which is to be compared with the bound (5.5).

It has been found that, for given $\beta, F, \epsilon$, and $\kappa^{2}$, the quantity given by (7.8) is maximized for the gravest meridional mode $m=1$. The dependence of (7.8) on $\kappa^{2}$, with $m=1$, is shown in Fig. 3 for various values of $\epsilon$, taking $\beta=10$ and $F=10$. Note that with $m=1$ we must have $\kappa^{2}>\pi^{2}$, since $\kappa^{2}=m^{2} \pi^{2}+k^{2}$ where $k$ is the zonal wavenumber; and, because of this, the instability is suppressed for sufficiently small $F$. It can be seen that as $\epsilon$ increases, the maximum shifts from the linearly most unstable wave $\kappa^{2}=\sqrt{ } 2 F$ to longer wavelengths, as is well known.

It may be noted here that the theory of Pedlosky (1970) leading to (7.3) breaks down at the point of minimum critical shear, $\kappa^{2}=\sqrt{ } 2 F$. The technical reasons for this are discussed by Pedlosky (1982a), where it is shown that the 1970 theory nevertheless remains self-consistent away from this point. The correct theory at minimum critical shear is given by Pedlosky (1982b). Numerical calculations by Boville (1981) suggest that, for $\epsilon=0.1$, the maximum amplitude $|A|^{2}$ at $\kappa^{2}=\sqrt{ } 2 F$ is underestimated by (7.3) by a factor of about twothirds. For sufficiently large $\epsilon$ however, Fig. 3 shows that the maximum is, in any case, attained well away from $\kappa^{2}=\sqrt{ } 2 F$, where (7.3) should be reasonably reliable.

If (7.8) is maximized over all $\kappa^{2}>\pi^{2}$, then the remaining parameters are $\beta, F$, and $\epsilon$. Inspection of (7.8) readily reveals that, for fixed $F$ and $\epsilon$, it has precisely the same dependence on $\beta$ as does the rigorous nonlinear bound (5.5), namely like $\beta^{2}$. On the other hand, variations in $\beta$ for fixed $U$ would imply variations in $\epsilon$. The other dependences of (7.8) are less obvious. Figure 4 compares the bound (5.5) over a range of $\epsilon$ with the maximum of (7.8), for various $F$. (Boville's 1981 maximum, for $F=8.7$, is also indicated on Fig. 4.) The agreement is seen to be quite close in many respects. While the bound (5.5) is strictly independent of $F$, the maximum of (7.8) is seen to have only a weak dependence on $F$. Moreover, the dependence on $\epsilon$ is rather similar in the two cases. [Note that for fixed $\kappa$ (7.8) increases linearly with $\epsilon$, as does (5.5).] Quantitatively, over its useful range of $\epsilon<1$, the rigorous 


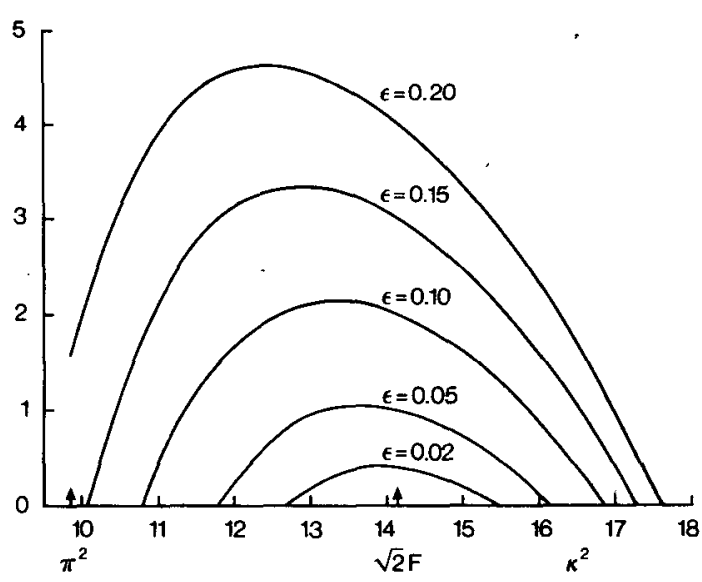

Fig. 3. Dependence of (7.8) on $\kappa^{2}$ for various values of the supercriticality $\epsilon$, taking $\beta=10, F=10$, and $m=1$. Equation (7.8) represents the maximum potential enstrophy of growing single waves according to Pedlosky's (1970) weakly nonlinear theory.

upper bound overestimates the maximum potential enstrophy from weakly nonlinear theory by less than a factor of two. For $\epsilon \geqslant 1$, it is evident that weakly nonlinear theory must be overestimating the wave amplitudes, as the maximum of (7.8) exceeds the total amount of potential enstrophy in the system, which suggests that the theory has become invalid. Indeed, at $O(1)$ supercriticality maximum wave amplitudes are known to be overestimated when wave-wave interactions are suppressed (Klein and Pedlosky 1986).

\section{Discussion}

This paper addresses the question of finite-amplitude saturation of baroclinic instability in a two-layer quasigeostrophic fluid. For an initial condition consisting of an unstable zonal flow with supercriticality $\epsilon$ and an infinitesimal wavy (i.e. nonzonal) disturbance, it has been shown that the potential enstrophy of the growing wave field is limited by a rigorous nonlinear bound, (4.17), which is approximately linear in $\epsilon$ for small $\epsilon$. A rigorous upper bound for the case of initial nonzonal disturbances of arbitrary magnitude is given by (4.10) with $\delta$ as in (4.12), and is shown in Fig. 1. These results are valid not only for conservative (unforced) flow, but also for forced-dissipative flow when the dissipation is proportional to the potential vorticity (section 6). In a comparison of the bound with the maximum wavy potential enstrophy predicted by Pedlosky's (1970) weakly nonlinear theory of single-wave equilibration for the Phillips model (section 7), the agreement was found to be quite close, with the nonlinear bound giving a value very similar to that obtained in weakly nonlinear theory.

In the case of the Phillips model (section 5), a suggestion by I. M. Held led to a significant improvement of the bound, viz., (5.5) as compared with (5.1). (It is of course a general difficulty with this method that one never knows whether a given bound could not be improved upon.) In this case, the improvement appears to depend on the stable basic state having $Q_{t}^{\prime}(y)$ constant within each layer. However, even with variable $Q_{i}^{\prime}(y)$ one may still use (5.3a) but replace (5.3b) with

$$
\begin{aligned}
& \int \frac{1}{2}\left\{\overline{q_{1}^{2}}+\overline{q_{2}^{2}}\right\}(t) d y \\
& \quad \leqslant \int \frac{1}{2}\left\{\frac{\left|Q_{1}^{\prime}(y)\right|_{\max }}{\left|Q_{1}^{\prime}(y)\right|_{\text {min }}} \overline{q_{1}^{2}}+\frac{\left|Q_{1}^{\prime}(y)\right|_{\text {max }}}{\left|Q_{2}^{\prime}(y)\right|_{\text {min }}} \overline{q_{2}^{2}}\right\}(0) d y .
\end{aligned}
$$

In the limit $\epsilon \rightarrow 0,\left|Q_{1}^{\prime}(y)\right|_{\max } /\left|Q_{1}^{\prime}(y)\right|_{\min }$ is $O(1)$ while $\left|Q_{1}^{\prime}(y)\right|_{\max } /\left|Q_{2}^{\prime}(y)\right|_{\min }$ is $\mathrm{O}\left(\epsilon^{-1}\right)$, so the second term on the right-hand side of (8.1) will dominate and one may expect to reduce the bounds obtained in section 4 by roughly a factor of two (as with the Phillips model). Indeed, by using (8.1) in place of (3.1) in this limit, the bound (4.10) will still apply but with $\Lambda$ given by the minimum of $1 / 2 \int G^{2} d y$ [instead of (4.7)], and $\hat{z}_{0}$ given by the initial eddy potential enstrophy in the lower layer alone [instead of (4.8)]. In any particular application, one would clearly be well advised to consider this sort of improvement to the bounds presented in section 4 .

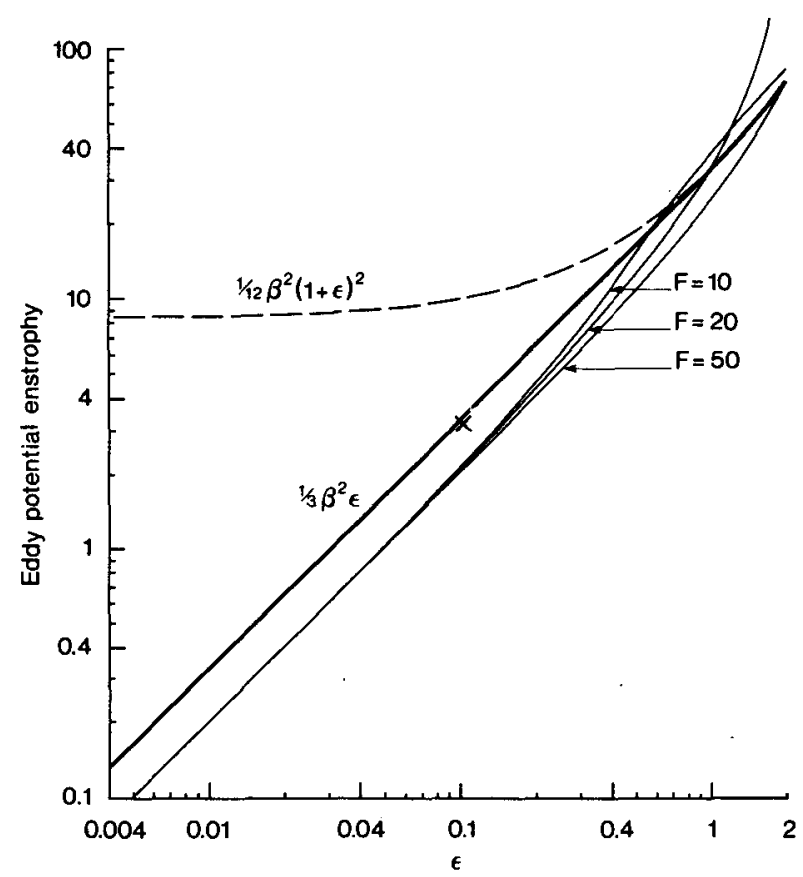

FIG. 4. Dependence on the supercriticality $\epsilon$ of the nonlinear bound (5.5) (thick solid curve); the total amount of potential enstrophy in the system (dashed curve); and the maximum over $\kappa^{2}$ of (7.8) for various values of $F$ (three thin solid curves), representing the maximum potential enstrophy for growing waves predicted by Pedlosky's (1970) weakly nonlinear theory. In all cases $\beta=10$. Boville's (1981) numerical calculation (with $F=8.7$ ) is indicated by a cross and has been corrected to account for the different value of $\beta$ that was used in his study. 
Although the derived bounds are on the potential enstrophy of the nonzonal flow, they can be turned into bounds on the energy by using the existence of a minimum wavenumber for nonzonal motions. To be precise, for the channel $0 \leqslant y \leqslant 1$ considered here one has

$$
\begin{aligned}
\int_{0}^{1} \frac{1}{2}\left\{\overline{\left|\nabla \psi_{1}^{\prime}\right|^{2}}+\overline{\left|\nabla \psi_{2}^{\prime}\right|^{2}}+\overline{F\left(\psi_{1}^{\prime}-\psi_{2}^{\prime}\right)^{2}}\right\} d y \\
\leqslant \pi^{-2} \int_{0}^{1} \frac{1}{2}\left\{\overline{q_{1}^{\prime 2}}+\overline{q_{2}^{\prime \prime}}\right\} d y
\end{aligned}
$$

(see Appendix), and therefore any bounds on the potential enstrophy [ $\pi^{2}$ times the right-hand side of (8.2)] imply bounds on the energy [the left-hand side of (8.2)] as well. A more accurate bound on the energy could be obtained in circumstances where something is known about the spatial structure of the eddies.

Several applications of these upper bounds come to mind. One is that they provide a useful constraint for evaluating the validity of approximate (e.g. weakly nonlinear or low-order) theories. For example, in the case of the Phillips model it was shown that the maximum potential enstrophy predicted by weakly nonlinear single-wave equilibration exceeds the rigorous nonlinear bound for $O(1)$ supercriticality (Fig. 4). The numerical experiments of Klein and Pedlosky (1986) in such a regime have indeed found that wave amplitudes are overestimated by the single-wave theory.

A second application is suggested by the fact that the bounds give parameter dependences for finiteamplitude saturation which (unlike those from weakly nonlinear theory) are unconstrained by any assumptions about the nature of the initial wavy disturbance. Because the bounds also appear to be quantitatively reasonable, this means that they might well provide a sound theoretical basis for obtaining eddy-amplitude closures in transient-eddy parameterization schemes. Naturally, it is important in this respect to determine just how accurate the bounds are as predictors of eddy amplitudes under fully nonlinear conditions, something which can only be decided by numerical simulation.

A fascinating question concerns the relationship between these results and geostrophic turbulence theories. At face value, at least, there is every possibility of a discrepancy because the turbulence theories explicitly neglect the material-conservation property of potential vorticity from which the generalized Charney-Stern theorem is derived. In the forced-dissipative case this argument loses its force; nevertheless, it would be interesting to study closure-model predictions under these conditions to see whether they were consistent with the rigorous bounds. For the case of conservative flow, to which the above argument is more relevant, there is (of course) no. quantitative turbulence theory for the continuous (as opposed to spectrally truncated) equations. However, one might imagine performing the usual kind of statistical-mechanical calculation for a given number of degrees of freedom, and then examining whether the fraction of potential enstrophy residing in the zonal flow tended to a limit as this number tended to infinity. If such a limit existed, it could then be compared with the minimum fraction permitted by the rigorous bounds. Statistical mechanics predicts an equipartition of potential enstrophy between all the modes, subject to the boundary conditions. Because the boundary conditions do not require that any of the baroclinic part of the potential enstrophy reside in the zonal flow (section 5), it therefore seems plausible that, at least for sufficiently small supercriticality, such a calculation would predict less potential enstrophy in the zonal flow than was actually permitted by the saturation bounds. It is clearly of interest to turn this conjecture into a more precise statement, but such a study is beyond the scope of the present paper.

It will be evident that the present approach is not restricted to the two-layer model. A generalized Charney-Stern theorem of the type (3.1) also exists in the case of continuously stratified flow (cf. McIntyre and Shepherd 1987, appendix B). In Part II of this study it will be used to derive nonlinear saturation bounds for the Charney model of baroclinic instability, as well as for other more realistic profiles.

Acknowledgments. The author wishes to thank Rick Salmon for suggesting that the methods of Shepherd (1987) might be illuminating in this context, Joe Pedlosky for helpful comments regarding the weakly nonlinear theory, Isaac Held for pointing out the improved bound in section 5, Peter Stone, Bill Gutowski and Peter Haynes for advice on presentation, and the anonymous referees. This research has been supported by the UK Natural Environment Research Council and by St. Cathrine's College, Cambridge.

\section{APPENDIX}

\section{Proof of (8.2)}

Let $\tau \equiv \Phi_{1}-\Phi_{2}$ be the baroclinic streamfunction, as before, and $\psi \equiv \Phi_{1}+\Phi_{2}$ the barotropic streamfunction (not to be confused with the disturbance streamfunction in each layer, $\psi_{i}$ ). Then using (2.4), the energy of the nonzonal flow, $E^{\prime}$ say, may be written

$$
E^{\prime}=\frac{1}{4}\left\{\left|\nabla \psi^{\prime}\right|^{2}+\left|\nabla \tau^{\prime}\right|^{2}\right\}+\frac{1}{2} F \tau^{\prime 2} .
$$

Similarly, using (2.5) the potential enstrophy of the nonzonal flow, $Z^{\prime}$ say, takes the form

$$
Z^{\prime}=\frac{1}{4}\left\{\left(\nabla^{2} \psi^{\prime}\right)^{2}+\left(\nabla^{2} \tau^{\prime}\right)^{2}\right\}+F^{2} \tau^{\prime 2}-F \tau^{\prime} \nabla^{2} \tau^{\prime}
$$

Now, consider the boundary-value problem posed by

$$
\nabla^{2} \phi+\kappa^{2} \phi=0
$$

in the channel domain, subject to the conditions

$$
\phi=0 \text { at } y=0,1
$$


[which is the relevant form of (2.3) for the nonzonal flow]. The minimum eigenvalue $\kappa$ for (A3) is evidently $\pi$. By expanding $\psi^{\prime}$ in terms of the eigenfunctions of (A3), noting that $\kappa^{2} \geqslant \pi^{2}$, and using the relation

$$
\int_{0}^{1} \overline{\left|\nabla \psi^{\prime}\right|^{2}} d y=-\int_{0}^{1} \overline{\psi^{\prime} \nabla^{2} \psi^{\prime}} d y
$$

[which follows from the boundary conditions (A3b)], one obtains

$$
\int_{0}^{1} \overline{\left(\nabla^{2} \psi^{\prime}\right)^{2}} d y \geqslant \pi^{2} \int_{0}^{1} \overline{\left|\nabla \psi^{\prime}\right|^{2}} d y
$$

Equation (A5) is just Poincaré's inequality for the channel, and evidently applies to $\tau^{\prime}$ as well. It remains to note that (A3a) directly implies

$$
-\tau^{\prime} \nabla^{2} \tau^{\prime} \geqslant \pi^{2} \tau^{\prime 2}
$$

then combining (A1), (A2), (A5) and (A6) yields

$$
\begin{aligned}
\int_{0}^{1} \overline{Z^{\prime}} d y & \geqslant \pi^{2} \int_{0}^{1} \frac{1}{4}\left\{\overline{\left|\nabla \psi^{\prime}\right|^{2}}+\overline{\left|\nabla \tau^{\prime}\right|^{2}}\right\} d y \\
& +2\left(F+\pi^{2}\right) \int_{0}^{1} \frac{1}{2} \overline{F \tau^{\prime 2}} d y \geqslant \pi^{2} \int_{0}^{1} \overline{E^{\prime}} d y,
\end{aligned}
$$

which is precisely equivalent to (8.2).

\section{REFERENCES}

Abarbanel, H. D. I., D. D. Holm, J. E. Marsden and T. Ratiu, 1986: Nonlinear stability analysis of stratified fluid equilibria. Phil. Trans. Roy. Soc. London, A318, 349-409.

Andrews, D. G., and M. E. McIntyre, 1976: Planetary waves in horizontal and vertical shear: The generalized Eliassen-Palm relation and the mean zonal acceleration. J. Atmos. Sci., 33, 2031-2048.

Arnol'd, V. I., 1966: On an a priori estimate in the theory of hydrodynamical stability. Izv. Vyssh. Uchebn. Zaved. Matematika, 54, no. 5, 3-5. [English transl.: Amer. Math. Soc. Transl., Series 2, 79, 267-269 (1969).]

Boville, B. A., 1981: Amplitude vacillation on a $\beta$-plane. J. Atmos. Sci., 38, 609-618.
Bronshtein, I. N., and K. A. Semendyayev, 1985: Handbook of Mathematics. Verlag Harri Deutsch and Van Nostrand Reinhold Company, $973 \mathrm{pp}$.

Charney, J. G., 1947: The dynamics of long waves in a baroclinic westerly current. J. Meteor., 4, 135-162.

- 1971: Geostrophic turbulence. J. Atmos. Sci., 28, 1087-1095.

Drazin, P. G., 1970: Non-linear baroclinic instability of a continuous zonal flow. Quart. J. Roy. Meteor. Soc., 96, 667-676.

Eady, E. T., 1949: Long waves and cyclone waves. Tellus, 1, 33-52.

Held, I. M., 1985: Pseudomomentum and the orthogonality of modes in shear flows. J. Atmos. Sci., 42, 2280-2288.

Herring, J. R., 1980: Statistical theory of quasi-geostrophic turbulence. J. Atmos. Sci., 37, 969-977.

Holm, D. D., J. E. Marsden, T. Ratiu and A. Weinstein, 1985: Nonlinear stability of fluid and plasma equilibria. Phys. Rep., 123, $1-116$.

Killworth, P. D., and M. E. McIntyre, 1985: Do Rossby-wave critical layers absorb, reflect or over-reflect? J. Fluid Mech., 161, 449492.

Klein, P., and J. Pedlosky, 1986: A numerical study of baroclinic instability at large supercriticality. J. Atmos. Sci., 43, 1243-1262.

McIntyre, M. E., and T. G. Shepherd, 1987: An exact local conservation theorem for finite-amplitude disturbances to non-parallel shear flows, with remarks on Hamiltonian structure and on Arnol'd's stability theorems. J. Fluid Mech., 181, 527-565.

Mak, M., 1985: Equilibration in nonlinear baroclinic instability. $J$. Atmos. Sci., 42, 2764-2782.

-, 1987: Dissipative structure of a nonlinear baroclinic system: Effect of asymmetric friction. J. Atmos. Sci., 44, 2613-2627.

Pedlosky, J., 1970: Finite-amplitude baroclinic waves. J. Atmos. Sci., 27, 15-30.

- 1979: Geophysical Fluid Dynamics. Springer-Verlag, 624 pp. 1982a: Finite-amplitude baroclinic waves at minimum critical shear. J. Atmos. Sci., 39, 555-562.

- 1982b: A simple model for nonlinear critical layers in an unstable baroclinic wave. J. Atmos. Sci., 39, 2119-2127.

Phillips, N. A., 1954: Energy transformations and meridional circulations associated with simple baroclinic waves in a two-level quasi-geostrophic model. Tellus, 6, 273-286.

Rhines, P. B., 1977: The dynamics of unsteady currents. The Sea, Vol. 6, E. D. Goldberg et al., Eds., Wiley 189-318.

Salmon, R., 1978: Two-layer quasi-geostrophic turbulence in a simple special case. Geophys. Astrophys. Fluid Dyn., 10, 25-52.

Shepherd, T. G., 1987: Non-ergodicity of inviscid two-dimensional flow on a beta-plane and on the surface of a rotating sphere. $J$. Fluid Mech., 184, 289-302.

- 1988: Rigorous bounds on the nonlinear saturation of instabilities to parallel shear flows. J. Fluid Mech., 195, in press. 